\title{
EQUIVARIANT COHOMOLOGY OF INFINITE-DIMENSIONAL GRASSMANNIAN AND SHIFTED SCHUR FUNCTIONS
}

\author{
JiA-Ming (Frank) Liou And Albert Schwarz
}

\begin{abstract}
We study the multiplication and comultiplication in the equivariant cohomology of Sato Grassmannian.
\end{abstract}

\section{Introduction}

Let us consider the Hilbert space $\mathcal{H}=L^{2}\left(S^{1}\right)$ and its subspaces $\mathcal{H}_{+}, \mathcal{H}_{-}$defined as closed subspaces of $\mathcal{H}$ spanned by $\left\{z^{i}: i \geq 0\right\}$ and $\left\{z^{j}: j<0\right\}$ respectively. Using the decomposition $\mathcal{H}=\mathcal{H}_{+} \oplus \mathcal{H}_{-}$one can define the (Segal-Wilson version of) Sato Grassmannian $\operatorname{Gr}(\mathcal{H})$ as a space of all closed linear subspaces $W \subset \mathcal{H}$ such that the projection $\pi_{-}: W \rightarrow \mathcal{H}_{-}$is a Fredholm operator and the projection $\pi_{+}: W \rightarrow \mathcal{H}_{+}$ is a compact operator (see [11] for more detail).

The group $S^{1}$ acts naturally on $\mathcal{H}$ : to every $\alpha$ obeying $|\alpha|=1$ we assign a map $f(z) \rightarrow f(\alpha z)$. This action (we will call it standard action) generates an action of $S^{1}$ on Grassmannian . Representing a function on a circle as a Fourier series $f(z)=\sum a_{n} z^{n}$, we see that the standard action sends $a_{k} \rightarrow \alpha^{k} a_{k}$. The standard action is related to the $S^{1}$-action on various moduli spaces embedded into Grassmannian by means of Krichever construction; see $[6,12]$ for more detail. Motivated by the desire to construct nonperturbative string theory we considered in [6] equivariant cohomology of Sato Grassmannian and homomorphism of this cohomology induced by the Krichever map. The results of the present paper will be used in [7] to study this homomorphism in more detail.

One can consider more general actions of $S^{1}$ on $\mathcal{H}$ sending $a_{k} \rightarrow \alpha^{n_{k}} a_{k}$ where $n_{k} \in \mathbb{Z}$ is an arbitrary doubly infinite sequence of integers. This action also generates an action of $S^{1}$ on Grassmannian. The Grassmannian $\operatorname{Gr}(\mathcal{H})$ is a disjoint union of connected components $\operatorname{Gr}_{d}(\mathcal{H})$ labeled by the index of the projection $\pi_{-}: W \rightarrow \mathcal{H}_{-}$. All components are homeomorphic. It was proven in [11] that every component is homotopy equivalent to a subspace having a cell decomposition $K=\cup \sigma_{\lambda}$ consisting of even-dimensional cells (finite-dimensional Schubert cells). The cells are labeled by partitions. This decomposition is $S^{1}$-invariant with respect to any action of $S^{1}$ from the class of actions we are interested in. This allows us to say that the equivariant cohomology $H_{S^{1}}\left(\mathrm{Gr}_{d}(\mathcal{H})\right)$ has a free system of generators $\Omega_{\lambda}^{T}=\left[\bar{\Sigma}_{\lambda}\right]$ as a module over $H_{S^{1}}$ (pt). These generators can be interpreted also as cohomology classes dual to Schubert cycles $\bar{\Sigma}_{\lambda}$ having finite codimension. In this paper, we calculate the multiplication table in the basis $\Omega_{\lambda}^{T}$.

Let $\mathbb{P}^{\infty}$ be the infinite-dimensional complex projective space and $\mathcal{O}(-1)$ be the tautological line bundle on $\mathbb{P}^{\infty}$. Let us denote $u=c_{1}(\mathcal{O}(-1))$, i.e., $u$ is the Chern

Received by the editors January 23, 2012. 
class of the tautological line bundle on $\mathbb{P}^{\infty}$. Since the $S^{1}$-equivariant cohomology of a point equals the conventional cohomology of $\mathbb{P}^{\infty}$, we identify $H_{S^{1}}^{*}(\mathrm{pt})$ with the polynomial ring $\mathbb{C}[u]$. Then, we have the following result:

Theorem 1.1. For the standard $S^{1}$-action on $\operatorname{Gr}_{d}(\mathcal{H})$ the coefficients in the decomposition

$$
\Omega_{\lambda}^{T} \Omega_{\mu}^{T}=\sum_{\nu} C_{\lambda \mu}^{\nu}(u) \Omega_{\nu}^{T}
$$

can be expressed in terms of coefficients in the decomposition

$$
s_{\lambda}^{*} s_{\mu}^{*}=\sum_{\nu} C_{\lambda \mu}^{\nu} s_{\nu}^{*}
$$

by the formula $C_{\lambda \mu}^{\nu}(u)=C_{\lambda \mu}^{\nu} u^{|\lambda|+|\mu|-|\nu|}$. Here $s_{\lambda}^{*}$ stands for the shifted Schur function in the sense of [10] and $|\lambda|=\sum_{i} \lambda_{i}$ is the weight of the partition $\lambda=\left(\lambda_{i}\right)$.

Combining this theorem with the results of [8], we obtain the following expression for the coefficients:

\section{Corollary 1.1.}

$$
C_{\lambda \mu}^{\nu}(u)=u^{|\lambda|+|\mu|-|\nu|} \sum_{\lambda, \mu \subset \rho \subset \nu}(-1)^{|\nu|-|\rho|} \frac{h(\rho)}{h(\nu / \rho) h(\rho / \lambda) h(\rho / \mu)} .
$$

Here the function $h$ is defined to be $h(\nu / \mu)=|\nu / \mu| ! / \operatorname{dim}(\nu / \mu)$ for any skew diagram $\nu / \mu$ and $\operatorname{dim}(\nu / \mu)$ is the number of standard $\nu / \mu$-tableaux.

Notice that the numbers $h(\nu / \mu)$ are not always integers but the numbers

$$
\sum_{\lambda, \mu \subset \rho \subset \nu}(-1)^{|\nu|-|\rho|} \frac{h(\rho)}{h(\nu / \rho) h(\rho / \lambda) h(\rho / \mu)}
$$

in (1.3) are non-negative integers. This follows immediately from Theorem 1.1. A combinatorial proof of integrality was given in [8] .

The Theorem 1.1 can be applied to the analysis of multiplication in tautological cohomology ring of the moduli spaces of complex curves (more precisely, to the study of intersection of cycles defined in terms of Weierstrass points); see [7]. To generalize the theorem to the non-standard action of $S^{1}$ we introduce the notion of shifted double Schur function (it is closely related to double Schur functions of infinite number of arguments introduced in [9]).

Let $x=\left(x_{1}, \ldots, x_{n}\right)$ be an $n$-tuple of variables and $y=\left(y_{i}\right)_{i \in \mathbb{Z}}$ be a doubly infinite sequence. Recall that, the double Schur function ${ }^{n} s_{\lambda}\left(x_{1}, \ldots, x_{n} \mid y\right)$ is ${ }^{1}$ a symmetric polynomial in $x=\left(x_{1}, \ldots, x_{n}\right)$ with coefficients in $\mathbb{C}[y]$ defined by

$$
{ }^{n} s_{\lambda}\left(x_{1}, \ldots, x_{n} \mid y\right)=\operatorname{det}\left[\left(x_{i} \mid y\right)^{\lambda_{j}+n-j}\right] / \operatorname{det}\left[\left(x_{i} \mid y\right)^{n-j}\right],
$$

where $\left(x_{i} \mid y\right)^{p}=\prod_{j=1}^{p}\left(x_{i}-y_{j}\right)$. Let us introduce the shift operator on $\mathbb{C}[y]$ given by

$$
(\tau y)_{i}=y_{i-1}, \quad i \in \mathbb{Z} .
$$

\footnotetext{
${ }^{1}$ We add the index $n$ to the conventional notation $s_{\lambda}$ to emphasize that the function depends on $n$ variables $x_{k}$.
} 
The double Schur function satisfies the generalized Jacobi-Trudi formula:

$$
{ }^{n} s_{\lambda}\left(x_{1}, \ldots, x_{n} \mid y\right)=\operatorname{det}\left[h_{\lambda_{i}+j-i}\left(x_{1}, \ldots, x_{n} \mid \tau^{j-1} y\right)\right]_{i, j=1}^{n},
$$

where

$$
h_{p}\left(x_{1}, \ldots, x_{n} \mid y\right)=\sum_{1 \leq i_{1} \leq \cdots \leq i_{p} \leq k}\left(x_{i_{1}}-y_{i_{1}}\right) \cdots\left(x_{i_{p}}-y_{i_{p}+p-1}\right), \quad p \geq 1 .
$$

For a proof of (1.4), the reader is referred to the page 56 of Macdonald's classical text [3].

The Littlewood-Richardson coefficients ${ }^{n} c_{\lambda \mu}^{\nu}(y)$ of the double Schur functions are defined by

$$
{ }^{n} s_{\lambda}\left(x_{1}, \ldots, x_{n} \mid y\right)^{n} s_{\mu}\left(x_{1}, \ldots, x_{n} \mid y\right)=\sum_{\nu}{ }^{n} c_{\lambda \mu}^{\nu}(y)^{n} s_{\mu}\left(x_{1}, \ldots, x_{n} \mid y\right) .
$$

These coefficients ${ }^{n} c_{\lambda \mu}^{\nu}(y)$ were calculated in [8]. We define the shifted double Schur function by

$$
{ }^{n} s_{\lambda}^{*}\left(x_{1}, \ldots, x_{n} \mid y\right)={ }^{n} s_{\lambda}\left(x_{1}+y_{-1}, x_{2}+y_{-2}, \ldots, x_{n}+y_{-n} \mid \tau^{n+1} y\right) .
$$

Under the change of variables $x_{i}^{\prime}=x_{i}+y_{-i}$ for $1 \leq i \leq n$, the shifted double Schur function ${ }^{n} s_{\lambda}^{*}\left(x_{1}, \ldots, x_{n} \mid y\right)$ becomes the double Schur function ${ }^{n} s_{\lambda}\left(x_{1}^{\prime}, \ldots, x_{n}^{\prime} \mid \tau^{n+1} y\right)$. Notice that the shifted double Schur function ${ }^{n} s_{\lambda}^{*}\left(x_{1}, \ldots, x_{n} \mid y\right)$ is the shifted Schur function defined in [10] if $y=\left(y_{k}\right)_{k}$ is the sequence defined by the relation $y_{k}=$ constant $+k$ for all $k$. The shifted $\mathrm{s}^{n} s_{\lambda}^{*}\left(x_{1}, \ldots, x_{n} \mid y\right)$ have the following stability property:

Proposition 1.1. If $l(\lambda)<n$, then

$$
{ }^{n+1} s_{\lambda}^{*}\left(x_{1}, \ldots, x_{n}, 0 \mid y\right)={ }^{n} s_{\lambda}^{*}\left(x_{1}, \ldots, x_{n} \mid y\right) .
$$

Here $l(\lambda)$ is the length of a partition $\lambda$.

Using this property, we can define the shifted double Schur function $s_{\lambda}^{*}(x \mid y)$ depending on infinite number of arguments $x=\left(x_{i}\right)_{i \in \mathbb{N}}$ and $y=\left(y_{j}\right)_{j \in \mathbb{Z}}$ (we assume that only finite number of variables $x_{i}$ does not vanish). Namely, we define

$$
s_{\lambda}^{*}(x \mid y)={ }^{n} s_{\lambda}^{*}\left(x_{1}, \ldots, x_{n} \mid y\right)
$$

where $n$ is chosen in such a way that $n>l(\lambda)$ and $x_{i}=0$ for $i>n$. The coefficients in the decomposition

$$
s_{\lambda}^{*}(x \mid y) s_{\mu}^{*}(x \mid y)=\sum C_{\lambda \mu}^{\nu}(y) s_{\nu}^{*}(x \mid y) .
$$

can be obtained from (1.5) and from the results of $[5,8,9]$. In fact, the relation between $C_{\lambda \mu}^{\nu}$ and ${ }^{n} c_{\lambda \mu}^{\nu}$ is given by

$$
C_{\lambda \mu}^{\nu}(y)={ }^{n} c_{\lambda \mu}^{\nu}\left(\tau^{n+1} y\right)
$$

for $n>l(\lambda), l(\mu), l(\nu)$.

The shifted double Schur functions belong to the ring $\Lambda^{*}(x \| y)$ that consists of polynomial functions that depend on sequences $x=\left(x_{n}\right)_{n \in \mathbb{N}}$ and $y=\left(y_{i}\right)_{i \in \mathbb{Z}}$ and are symmetric with respect to shifted variables $x_{i}^{\prime}=x_{i}+y_{-i}$ (we assume that $x_{n}=0$ 
for $n \gg 0) .^{2}$ Moreover, they form a free system of generators of $\Lambda^{*}(x \| y)$ considered as $\mathbb{C}[y]$-module. Note that the ring $\Lambda^{*}(x \| y)$ is obviously isomorphic to the ring $\Lambda(x \| y)$ constructed in [9]. The following theorem describes the multiplication in the equivariant cohomology $H_{S^{1}}\left(\operatorname{Gr}_{d}(\mathcal{H})\right)$ for non-standard action of $S^{1}$ :

Theorem 1.2.

$$
\Omega_{\lambda}^{T} \Omega_{\mu}^{T}=\sum_{\nu} C_{\lambda \mu}^{\nu}(n) \Omega_{\nu}^{T}
$$

where $C_{\lambda \mu}^{\nu}(n)$ are the coefficients in (1.8) calculated for $y_{k}=n_{k+d} u$ and $n_{k}$ denotes the sequence specifying the action of $S^{1}$.

The Theorem 1.1 is a particular case of Theorem 1.2 for $n_{k}=k$.

Let us consider the infinite-dimensional torus $\mathbb{T}$ and its action on the Grassmannian. Algebraically the infinite torus $\mathbb{T}$, is the infinite direct product $\prod_{i \in \mathbb{Z}} S^{1}$. The action of $\mathbb{T}$ on Grassmannian corresponds to the action on $\mathcal{H}$ transforming $a_{k} \rightarrow \alpha_{k} a_{k}$, where $\left(\alpha_{k}\right) \in \mathbb{T}$ and $f=\sum_{n} a_{n} z^{n} \in \mathcal{H}$. This action specifies an embedding of $\mathbb{T}$ into the group of unitary transformations of $\mathcal{H}$; the topology of $\mathbb{T}$ is induced by this embedding.

One can prove that the equivariant cohomology $H_{\mathbb{T}}(p t)$ (cohomology of the classifying space $\left.B_{\mathbb{T}}\right)$ is isomorphic to the polynomial ring $\mathbb{C}[\mathbf{u}]$ where $\mathbf{u}$ stands for the doubly infinite sequence $u_{k}$. The proof is based on the consideration of homomorphisms of $\mathbb{T}$ onto finite-dimensional tori and homomorphisms of $S^{1}$ into $\mathbb{T}$. The finite codimensional Schubert cells are $\mathbb{T}$-invariant and hence the equivariant cohomology $H_{\mathbb{T}}\left(\mathrm{Gr}_{d}(\mathcal{H})\right)$ is a free $H_{\mathbb{T}}(p t)$-module generated by cohomology classes labeled by partitions; we denote these classes by $\Omega_{\lambda}^{T}$.

The submanifold $\mathrm{Gr}_{d}^{l}$ of $\mathrm{Gr}_{d}(\mathcal{H})$ consisting of points $W$ so that the orthogonal projection $\pi_{l}: W \rightarrow z^{-l} \mathcal{H}_{-}$is surjective. The intersection of Schubert cycle $\bar{\Sigma}_{\lambda}$ and $\operatorname{Gr}_{d}^{l}$ is denoted by $\bar{\Sigma}_{\lambda, l}$. The equivariant cohomology class in $H_{\mathbb{T}}^{*}\left(\operatorname{Gr}_{d}^{l}\right)$ corresponding to $\bar{\Sigma}_{\lambda, l}$ is denoted by $\Omega_{\lambda, l}^{T}$. The Schubert cycle $\bar{\Sigma}_{\lambda}$ and $\operatorname{Gr}_{d}^{l}$ are in general position if $l(\lambda)<d+l$; then we have

$$
f_{l}^{*} \Omega_{\lambda}^{T}=\Omega_{\lambda, l}^{T}
$$

where $f_{l}^{*}: H_{\mathbb{T}}^{*}\left(\operatorname{Gr}_{d}(\mathcal{H})\right) \rightarrow H_{\mathbb{T}}^{*}\left(\operatorname{Gr}_{d}^{l}\right)$ is map induced by the inclusion map $f_{l}: \mathrm{Gr}_{d}^{l} \rightarrow$ $\operatorname{Gr}_{d}(\mathcal{H})$.

The classes $\Omega_{\lambda, l}^{T}$ for $l(\lambda)<d+l$ form an additive system of generators of equivariant cohomology. It follows from (1.5) that

Theorem 1.3. The multiplication table in $H_{\mathbb{T}}^{*}\left(\mathrm{Gr}_{d}^{l}\right)$ is given by

$$
\Omega_{\lambda, l}^{T} \Omega_{\mu, l}^{T}=\sum_{\nu}{ }^{d+l} c_{\lambda \mu}^{\nu}(y) \Omega_{\nu, l}^{T},
$$

where $y=\left(y_{i}\right)_{i \in \mathbb{Z}}$ is the sequence defined by $y=\tau^{l+1} \mathbf{u}$.

Using the coefficients in (1.8), we can calculate the multiplication table in equivariant cohomology of Grassmannian $\mathrm{Gr}_{d}(\mathcal{H})$ with respect to the infinite torus action. Namely,

\footnotetext{
${ }^{2}$ Not very rigorously one can say that the ring $\Lambda(x \| y)$ is the ring of functions of $x$ and $y$ that are symmetric with respect to $x$ and become polynomial after the shift of variables.
} 
Theorem 1.4. The multiplication table in $H_{\mathbb{T}}^{*}\left(\operatorname{Gr}_{d}(\mathcal{H})\right)$ is given by:

$$
\Omega_{\lambda}^{T} \Omega_{\mu}^{T}=\sum_{\nu} C_{\lambda \mu}^{\nu}(y) \Omega_{\nu}^{T}
$$

where $\left(y_{k}\right)$ is the sequence given by the relation $y=\tau^{-d} \mathbf{u}$. The coefficient in this equation comes from the formula (1.8); it is considered as an element of $H_{\mathbb{T}}(p t)$.

Using homomorphisms of $S^{1}$ into $\mathbb{T}$ one can derive this theorem from Theorem 1.2 (conversely, one can deduce Theorem 1.2 from Theorem 1.4). It follows from Theorem 1.4 that the equivariant cohomology $H_{\mathbb{T}}^{*}\left(\mathrm{Gr}_{d}(\mathcal{H})\right)$ is isomorphic to the ring $\Lambda^{*}(x \| y)$.

We can introduce a comultiplication in the ring $H_{\mathbb{T}}^{*}\left(\operatorname{Gr}_{d}(\mathcal{H})\right)$ using the map

$$
\rho: \operatorname{Gr}_{0}\left(\mathcal{H}^{\prime}\right) \times \operatorname{Gr}_{0}\left(\mathcal{H}^{\prime \prime}\right) \rightarrow \operatorname{Gr}_{0}\left(\mathcal{H}^{\prime} \oplus \mathcal{H}^{\prime \prime}\right)
$$

defined by $(V, W) \mapsto V \oplus W$. Namely, we take $\mathcal{H}^{\prime}=\mathcal{H}_{\text {even }}$ (the subspace spanned by $z^{2 k}$ ) and $\mathcal{H}^{\prime \prime}=\mathcal{H}_{\text {odd }}$ (the space spanned by $z^{2 k+1}$ ). Then $\mathcal{H}^{\prime} \oplus \mathcal{H}^{\prime \prime}=\mathcal{H}$ and the map $\rho$ determines a homomorphism of $\mathbb{T}$-equivariant cohomology

$$
H_{\mathbb{T}}\left(\operatorname{Gr}_{0}(\mathcal{H})\right) \rightarrow H_{\mathbb{T}}\left(\operatorname{Gr}_{0}\left(\mathcal{H}_{\text {even }}\right) \times \operatorname{Gr}_{0}\left(\mathcal{H}_{\text {odd }}\right)\right)
$$

It is easy to prove that $H_{\mathbb{T}}\left(\operatorname{Gr}_{0}\left(\mathcal{H}_{\text {even }}\right) \times \operatorname{Gr}_{0}\left(\mathcal{H}_{\text {odd }}\right)\right)$ is isomorphic to tensor product of two copies of $H_{\mathbb{T}}^{*}\left(\operatorname{Gr}_{0}(\mathcal{H})\right)$; we obtain a comultiplication $\Delta$ in $H_{\mathbb{T}}^{*}\left(\operatorname{Gr}_{0}(\mathcal{H})\right)$.

Let us introduce the notion of the $k$ th shifted power sum function in $\Lambda^{*}(x \| y)$ :

$$
p_{k}(x \mid y)=\sum_{i=1}^{\infty}\left[\left(x_{i}+y_{-i}\right)^{k}-y_{-i}^{k}\right] \text {. }
$$

Here, we assume that $x_{n}=0$ for $n \gg 0$; hence (1.10) is a finite sum. We will prove that

$$
\Delta p_{k}=p_{k} \otimes 1+1 \otimes p_{k}, \quad k \geq 1 .
$$

In other words, we have the following theorem:

Theorem 1.5. The comultiplication $\Delta$ in the ring $H_{\mathbb{T}}^{*}\left(\operatorname{Gr}_{d}(\mathcal{H})\right)$ defined above coincides with the comultiplication in the ring $\Lambda^{*}(x \| y)=\Lambda(x \| y)$ constructed in [9].

\section{Equivariant Schubert classes}

The Grassmannian $\operatorname{Gr}(\mathcal{H})$ has a stratification in terms of Schubert cells having finite codimension; it is a disjoint union of $\mathbb{T}$-invariant submanifolds $\Sigma_{S}$ labeled by $S$, where $S$ is a subset of $\mathbb{Z}$ such that the symmetric difference $\mathbb{Z}_{-} \Delta S$ is a finite set. The Schubert cells $\Sigma_{S}$ are in one-to-one correspondence with the $\mathbb{T}$-fixed points $\mathcal{H}_{S}$, where $\mathcal{H}_{S}$ is the closed subspace of $\mathcal{H}$ spanned by $\left\{z^{s}: s \in S\right\}$ (the fixed point $\mathcal{H}_{S}$ is contained in the Schubert cell $\Sigma_{S}$ ). Instead of a subset $S$ of $\mathbb{Z}$, we can consider a decreasing sequence $\left(s_{n}\right)_{n}$ of integers. It is easy to check that $s_{n}=-n+d$ for $n \gg 0$, where $d$ is the index of $\mathcal{H}_{S}$. The complex codimension of the Schubert cell $\Sigma_{S}$ is given by the formula

$$
\operatorname{codim} \Sigma_{S}=\sum_{i=1}^{\infty}\left(s_{i}+i-d\right) .
$$

The closure $\bar{\Sigma}_{S}$ of $\Sigma_{S}$ is called the Schubert cycle of characteristic sequence $S$. It defines a cohomology class in $H^{*}(\operatorname{Gr}(\mathcal{H}))$ having dimension equal to $2 \operatorname{codim} \Sigma_{S}$. Since 
the Schubert cycle $\bar{\Sigma}_{S}$ is $\mathbb{T}$-invariant, it specifies also an element $\Omega_{S}^{T}$ in $H_{\mathbb{T}}^{*}(\operatorname{Gr}(\mathcal{H}))$. Denote $\lambda_{n}=s_{n}+n-d$ for $n \geq 1$. Then $\left(\lambda_{n}\right)$ form a partition. Instead of using the sequence $S$ to label the (equivariant) cohomology class $\Omega_{S}^{T}$, we use the notation $\Omega_{\lambda}^{T}$. Then the dimension of $\Omega_{\lambda}^{T}$ is equal to $2|\lambda|$. Similarly, we denote $\Sigma_{S}$ by $\Sigma_{\lambda}$. For more details see $[6,11]$.

Let us consider the submanifold $\operatorname{Gr}_{d}^{l}$ consisting of points $W$ in $\operatorname{Gr}_{d}(\mathcal{H})$ such that the orthogonal projection $\pi_{l}: W \rightarrow z^{-l} \mathcal{H}_{-}$is surjective. There is an equivariant vector bundle of rank $n=d+l$ over $\operatorname{Gr}_{d}^{l}$ whose fiber over $W$ is the kernel of the projection $\pi_{l}: W \rightarrow z^{-1} \mathcal{H}_{-}$. The action of $\mathbb{T}$ (or any action of $S^{1}$ ) on $\operatorname{Gr}_{d}(\mathcal{H})$ induces an action on $\operatorname{Gr}_{d}^{l}$. The equivariant Schubert cycle $\bar{\Sigma}_{\lambda, l}$ in $\operatorname{Gr}_{d}^{l}$ is the intersection of the equivariant Schubert cycle $\bar{\Sigma}_{\lambda}$ in $\operatorname{Gr}_{d}(\mathcal{H})$ and $\operatorname{Gr}_{d}^{l}$. The dual equivariant cohomology class of $\bar{\Sigma}_{\lambda, l}$ in $H_{\mathbb{T}}^{*}\left(\operatorname{Gr}_{d}^{l}\right)$ is denoted by $\Omega_{\lambda, l}^{T}$. If $l(\lambda)<d+l$ where $l(\lambda)$ means the length of the partition $\lambda$ then the Schubert cycle $\bar{\Sigma}_{\lambda}$ and $\mathrm{Gr}_{d}^{l}$ are in general position; hence we have

$$
f_{l}^{*} \Omega_{\lambda}^{T}=\Omega_{\lambda, l}^{T}
$$

where $f_{l}^{*}: H_{\mathbb{T}}^{*}\left(\operatorname{Gr}_{d}(\mathcal{H})\right) \rightarrow H_{\mathbb{T}}^{*}\left(\operatorname{Gr}_{d}^{l}\right)$ is the homomorphism induced by the inclusion map $f_{l}: \mathrm{Gr}_{d}^{l} \rightarrow \operatorname{Gr}_{d}(\mathcal{H})$.

Proposition 2.1. The equivariant Schubert class $\Omega_{\lambda, l}^{T}$ in $\operatorname{Gr}_{d}(\mathcal{H})$ is given by

$$
\Omega_{\lambda, l}^{T}=\operatorname{det}\left[c_{\lambda_{i}+j-i}^{T}\left(\underline{\mathcal{H}}_{-l, \lambda_{i}-i+d-1}-\mathcal{E}_{l}\right)\right]_{i, j=1}^{n} .
$$

Here $\underline{\mathcal{H}}_{i, j}$ is the equivariant vector bundle $\mathcal{H}_{i, j} \times \operatorname{Gr}_{d}^{l}$ and the vector space $\mathcal{H}_{i, j}$ is the subspace of $\mathcal{H}$ spanned by $\left\{z^{k}: i \leq k \leq j\right\}$.

Proof. This statement follows from the Kempf-Laksov's formula; see $[1,4]$.

\section{Structure constants of schubert classes with respect to the standard $S^{1}$-action}

Let $\mathcal{H}_{S}$ be a fixed point of $\mathbb{T}$-action on $\operatorname{Gr}_{d}(\mathcal{H})$. The inclusion map $\iota_{S}:\left\{\mathcal{H}_{S}\right\} \rightarrow$ $\operatorname{Gr}_{d}(\mathcal{H})$ induces a homomorphism:

$$
\iota_{S}^{*}: H_{S^{1}}^{*}\left(\operatorname{Gr}_{d}(\mathcal{H})\right) \rightarrow H_{S^{1}}^{*}\left(\left\{\mathcal{H}_{S}\right\}\right)
$$

called the restriction map. Denote by $\delta=\left(\delta_{i}\right)$ the partition corresponding to $S$, i.e., $\delta_{i}=s_{i}+i-d$. Assume that $\lambda=\left(\lambda_{i}\right)$ is a partition such that $l(\lambda)<l(\delta)$. Then

\section{Lemma 3.1.}

$$
\iota_{S}^{*} \Omega_{\lambda, l}^{T}=u^{|\lambda|} s_{\lambda}^{*}\left(\delta_{1}, \ldots, \delta_{d+l}\right) .
$$

Here $s_{\lambda}^{*}$ is the shifted Schur function defined in [10].

Proof. Denote $n=d+l$. Assume that $x_{1}^{\prime}, \ldots, x_{n}^{\prime}$ are equivariant Chern roots of $\mathcal{E}_{l}^{\vee}$ (the dual equivariant vector bundle of $\left.\mathcal{E}_{l}\right)$. Then $c^{T}\left(\mathcal{E}_{l}\right)=\prod_{i=1}^{n}\left(1-x_{i}^{\prime}\right)$. We also have $c^{T}\left(\underline{\mathcal{H}}_{-l, m}\right)=\prod_{j=-l}^{m}(1-j u)$. This gives us: for each $p \geq 0$,

$$
c_{p+j-i}^{T}\left(\underline{\mathcal{H}}_{-l, \lambda_{i}-i+d-1}-\mathcal{E}_{l}\right)=\sum_{a+b=p+j-i} h_{a}\left(x_{1}^{\prime}, \ldots, x_{n}^{\prime}\right)(-1)^{b} e_{b}\left(y_{-n}, y_{-n+1}, \ldots, y_{\lambda_{i}-i-1}\right)
$$

where $y_{j}=(j+d) u$ for $j \in \mathbb{Z}$. By the generalized Jacobi-Trudi formula (1.4), we find

$$
\Omega_{\lambda, l}^{T}={ }^{n} s_{\lambda}\left(x_{1}^{\prime}, \ldots, x_{n}^{\prime} \mid \tau^{n+1} y\right) .
$$


Applying the restriction homomorphism $\iota_{S}^{*}$, we find

$$
\iota_{S}^{*} \Omega_{\lambda, l}^{T}={ }^{n} s_{\lambda}\left(\delta_{1}-1+d, \ldots, \delta_{n}-n+d \mid \tau^{n+1} a\right) u^{|\lambda|}={ }^{n} s_{\lambda}^{*}\left(\delta_{1}, \ldots, \delta_{n} \mid a\right) u^{|\lambda|},
$$

where $a=\left(a_{j}\right)$ with $a_{j}=j+d$ for all $j \in \mathbb{Z}$. The shifted double Schur function ${ }^{n} s_{\lambda}^{*}\left(\delta_{1}, \ldots, \delta_{n} \mid a\right)$ coincides with the shifted Schur function defined in [10]. This completes the proof.

Taking the limit $l \rightarrow \infty$ we obtain

$$
\iota_{S}^{*} \Omega_{\lambda}^{T}=s_{\lambda}^{*}\left(\delta_{1}, \ldots, \delta_{k}, \ldots \mid a\right) u^{|\lambda|},
$$

Now we are ready to prove the Theorem 1.1. Since $C_{\lambda \mu}^{\nu}(u)$ is of the form $C_{\lambda \mu}^{\nu}$ $u^{|\lambda|+|\mu|-|\nu|},(1.1)$ can be rewritten as

$$
\Omega_{\lambda}^{T} \Omega_{\mu}^{T}=\sum_{\nu} C_{\lambda \mu}^{\nu} u^{|\lambda|+|\mu|-|\nu|} \Omega_{\nu}^{T}
$$

Applying $\iota_{S}^{*}$ to the above equation ${ }^{3}$ and using Lemma 3.1, we find that the constants $\left(C_{\lambda \mu}^{\nu}\right)$ satisfy $(1.2)$.

In order to compute the structure constants of the equivariant cohomology of $\operatorname{Gr}_{d}(\mathcal{H})$ relative to the basis consisting of the equivariant Schubert classes, we have to introduce the notion of shifted symmetric functions.

\section{Algebra of shifted symmetric functions}

Let $x=\left(x_{n}\right)_{n \in \mathbb{N}}$ be a sequence of variables obeying $x_{n}=0$ for $n \gg 0$ and $y=\left(y_{i}\right)_{i \in \mathbb{Z}}$ be a doubly infinite sequence of variables. Denote the set of pairs $(x, y)$ by $R$. Let us consider a function $f(x \mid y)$ such that its restriction $f_{n}$ to the subset $R_{k}$ specified by the condition $x_{k+1}=x_{k+2}=\cdots=0$ is a polynomial for every $k \in \mathbb{N}$. We say that $f$ is shifted symmetric if $f_{n}$ symmetric with respect to the variables $x_{i}^{\prime}=x_{i}+y_{-i}$ for $1 \leq i \leq n$. In other words,

$$
f_{n}^{\prime}\left(x_{1}^{\prime}, \ldots, x_{n}^{\prime} \mid y\right)=f_{n}\left(x_{1}^{\prime}-y_{-1}, \ldots, x_{n}^{\prime}-y_{-n} \mid y\right)
$$

is symmetric with respect to $x^{\prime}=\left(x_{1}^{\prime}, \ldots, x_{n}^{\prime}\right)$.

This definition is motivated by the definition in [10]. (If we replace $y_{j}$ by constant + $j$, we obtain the definition of the shifted symmetric functions given in [10].) An essentially equivalent notion was introduced in [9]. Instead of $R$ one can consider a set $\tilde{R}$ of pairs $(x, y)$ where $x=\left(x_{n}\right)_{n \in \mathbb{N}}$ and $y=\left(y_{i}\right)_{i \in \mathbb{Z}}$ are sequences obeying $x_{n}=y_{-n}$ for $n \gg 0$. Shifted symmetric functions on $R$ correspond to symmetric functions on $\tilde{R}$; this correspondence can be used to relate our approach to the approach of [9]. It is obvious that shifted symmetric functions on $R$ constitute a ring; we denote this ring by $\Lambda^{*}(x \| y)$. It is clear that this ring is isomorphic to the ring $\Lambda(x \| y)$ of symmetric functions on $\tilde{R}$ considered in [9]. It follows immediately from well known results that the shifted double Schur functions $\left\{s_{\lambda}^{*}(x \mid y)\right\}$ form a linear basis for $\Lambda^{*}(x \| y)$ considered as $\mathbb{C}[y]$-module.

Now we are ready to finish the proof of Theorem 1.4. Let $\left\{x_{1}^{\prime}, \ldots, x_{n}^{\prime}\right\}$ be the equivariant Chern roots of of the equivariant vector bundle $\mathcal{E}_{l}^{\vee}$. Denote $\mathbf{u}=\left(u_{i}\right)_{i \in \mathbb{Z}}$ the sequence of weights of the action of $\mathbb{T}$ on $\mathcal{H}$ and $y=\tau^{-d} \mathbf{u}$. Define $x_{i}=x_{i}^{\prime}-y_{-i}$ for $1 \leq i \leq n$.

\footnotetext{
${ }^{3}$ Here we may assume that $l(\lambda), l(\mu), l(\nu)<l(\delta)$. If $l(\lambda)>l(\delta), \iota_{S}^{*} \Omega_{\lambda}^{T}=0$.
} 
Theorem 4.1. The equivariant Schubert class $\Omega_{\lambda, l}^{T}$ is given by

$$
\Omega_{\lambda, l}^{T}={ }^{d+l} s_{\lambda}^{*}\left(x_{1}, \ldots, x_{d+l} \mid y\right) .
$$

Proof. The proof is the same as that in Lemma 3.1 .

This theorem allows us to define the algebra isomorphism between the equivariant cohomology ring $H_{\mathbb{T}}^{*}\left(\mathrm{Gr}_{d}\right)$ and the algebra of shifted symmetric functions $\Lambda^{*}(x \| y)$. The isomorphism between them is given by

$$
\Omega_{\lambda}^{T} \mapsto s_{\lambda}^{*}(x \mid y) .
$$

This implies that the multiplication table in $H_{T}^{*}\left(\operatorname{Gr}_{d}(\mathcal{H})\right)$ with respect to the basis $\left\{\Omega_{\lambda}^{T}\right\}$ is the same as the multiplication table of $\left\{s_{\lambda}^{*}(x \mid y)\right\}$ in $\Lambda^{*}(x \| y)$. This completes the proof of the Theorem 1.4.

\section{Comultiplication}

Our main goal in this section is to prove Theorem 1.5. Since the algebra of shifted symmetric functions $\Lambda^{*}(x \| y)$ is isomorphic to the algebra of symmetric functions $\Lambda(x \| y)$ with an isomorphism given by the change of variables, we will use $\Lambda(x \| y)$ for convenience. Without loss of generality, we may assume that all indices $d$ are zero, i.e., we consider the product map

$$
\rho: \operatorname{Gr}_{0}\left(\mathcal{H}_{\text {even }}\right) \times \operatorname{Gr}_{0}\left(\mathcal{H}_{\text {odd }}\right) \rightarrow \operatorname{Gr}_{0}(\mathcal{H}) .
$$

The infinite torus acts on $\mathcal{H}$ and thus acts on $\mathcal{H}_{\text {even }}$ and $\mathcal{H}_{\text {odd }}$ in a natural way; the map $\rho$ is equivariant. Since the Grassmannian is equivariantly formal (see $[1,2]$ ) we obtain that

$$
H_{\mathbb{T}}^{*}\left(\operatorname{Gr}_{0}(\mathcal{H})\right)=H_{\mathbb{T}}^{*}(p t) \otimes H^{*}\left(\operatorname{Gr}_{0}(\mathcal{H})\right)
$$

as a $H_{\mathbb{T}}^{*}(p t)$-module. Using the Künneth theorem and relation $\mathbb{C}[\mathbf{u}]=\mathbb{C}\left[\mathbf{u}_{\text {even }}\right] \otimes$ $\mathbb{C}\left[\mathbf{u}_{\text {odd }}\right]$, we find that

$$
H_{\mathbb{T}}^{*}\left(\operatorname{Gr}\left(\mathcal{H}_{\text {even }}\right) \times \operatorname{Gr}\left(\mathcal{H}_{\text {odd }}\right)\right)=H_{\mathbb{T}}^{*}\left(\operatorname{Gr}\left(\mathcal{H}_{\text {even }}\right)\right) \otimes H_{\mathbb{T}}^{*}\left(\operatorname{Gr}\left(\mathcal{H}_{\text {odd }}\right)\right)
$$

as $H_{\mathbb{T}}^{*}(p t)$-modules, where $\mathbf{u}_{\text {even }}=\left(u_{2 k}\right)_{k \in \mathbb{Z}}$ and $\mathbf{u}_{\text {odd }}=\left(u_{2 k+1}\right)_{k \in \mathbb{Z}}$.

For each $l \geq 1$, let $\operatorname{Gr}_{0}^{l}\left(\mathcal{H}_{\text {even }}\right)$ and $\operatorname{Gr}_{0}^{l}\left(\mathcal{H}_{\text {odd }}\right)$ be respectively the submanifolds of $\operatorname{Gr}_{0}\left(\mathcal{H}_{\text {even }}\right)$ and $\operatorname{Gr}_{0}\left(\mathcal{H}_{\text {odd }}\right)$ consisting of points $W$ so that the orthogonal projection $\pi_{\text {even }, l}: W \rightarrow z^{-2[l / 2]} \mathcal{H}_{\text {even, }-}$ is surjective if $W \in \operatorname{Gr}_{0}\left(\mathcal{H}_{\text {even }}\right)$ and $\pi_{\text {odd }, l}: W \rightarrow$ $z^{-2[l / 2]-2} \mathcal{H}_{\text {odd,- }}$ is surjective if $W \in \mathrm{Gr}_{0}\left(\mathcal{H}_{\text {odd }}\right)$. We can restrict the equivariant map (1.9) to the equivariant map

$$
\rho: \operatorname{Gr}_{0}^{l}\left(\mathcal{H}_{\text {even }}\right) \times \operatorname{Gr}_{0}^{l}\left(\mathcal{H}_{\text {odd }}\right) \rightarrow \operatorname{Gr}_{0}^{l} .
$$

Then the pullback bundle $\rho^{*} \mathcal{E}_{l}$ over $\operatorname{Gr}_{0}^{l}\left(\mathcal{H}_{\text {even }}\right) \times \operatorname{Gr}_{0}^{l}\left(\mathcal{H}_{\text {odd }}\right)$ splits into the direct sum of $\mathbb{T}$-equivariant vector bundles $\mathcal{E}_{l \text {,even }}$ and $\mathcal{E}_{l \text {,odd }}$, where the vector bundle $\mathcal{E}_{l \text {,even }}$ and $\mathcal{E}_{l, \text { odd }}$ are respectively the $\mathbb{T}$-equivariant bundle over $\operatorname{Gr}_{0}^{l}\left(\mathcal{H}_{\text {even }}\right)$ and over $\left.\operatorname{Gr}_{0}^{l}\left(\mathcal{H}_{\text {odd }}\right)\right)$ whose fiber over $W$ is the kernel of the orthogonal projection

$$
\pi_{\text {even }, l}: W \rightarrow z^{-2[l / 2]} \mathcal{H}_{\text {even, }-}
$$

if $W \in \operatorname{Gr}_{0}^{l}\left(\mathcal{H}_{\text {even }}\right)$ and is the kernel of

$$
\pi_{\text {odd }, l}: W \rightarrow z^{-2[l / 2]-2} \mathcal{H}_{\text {odd },-}
$$


if $W \in \operatorname{Gr}_{0}^{l}\left(\mathcal{H}_{\text {odd }}\right)$. Analogously, the $\mathbb{T}$-equivariant vector bundle $\rho^{*} \underline{\mathcal{H}}_{-l,-1}$ over $\operatorname{Gr}_{0}^{l}\left(\mathcal{H}_{\text {even }}\right) \times \operatorname{Gr}_{0}^{l}\left(\mathcal{H}_{\text {odd }}\right)$ splits into a direct sum of $\mathbb{T}$-equivariant bundles $\mathcal{F}_{l \text {,even }}$ and $\mathcal{F}_{l, \text { odd }}$, where $\mathcal{F}_{l \text {,even }}$ and $\mathcal{F}_{l \text {,odd }}$ are the product bundles of the form $F_{\text {even }} \times$ $\left(\operatorname{Gr}_{0}^{l}\left(\mathcal{H}_{\text {even }}\right) \times \operatorname{Gr}_{0}^{l}\left(\mathcal{H}_{\text {odd }}\right)\right)$ and $F_{\text {odd }} \times\left(\operatorname{Gr}_{0}^{l}\left(\mathcal{H}_{\text {even }}\right) \times \operatorname{Gr}_{0}^{l}\left(\mathcal{H}_{\text {odd }}\right)\right) ; F_{l \text {,even }}$ and $F_{l, \text { odd }}$ are the linear spaces spanned by $\left\{z^{2 s}:-l \leq 2 s \leq-1\right\}$ and by $\left\{z^{2 s+1}:-l \leq 2 s+1 \leq-1\right\}$, respectively. In other words, we have

$$
\rho^{*} \mathcal{E}_{l}=\mathcal{E}_{l, \text { even }} \oplus \mathcal{E}_{l, \text { odd }} \quad \text { and } \quad \rho^{*} \underline{\mathcal{H}}_{-l,-1}=\mathcal{F}_{l, \text { even }} \oplus \mathcal{F}_{l, \text { odd }}
$$

as $\mathbb{T}$-equivariant vector bundles over $\operatorname{Gr}_{0}^{l}\left(\mathcal{H}_{\text {even }}\right) \times \operatorname{Gr}_{0}^{l}\left(\mathcal{H}_{\text {odd }}\right)$.

Assume that $x_{1}, \ldots, x_{l}$ are the equivariant Chern roots of $\mathcal{E}_{l}$ and define the power sum functions $p_{k}\left(x_{1}, \ldots, x_{l} \mid \mathbf{u}\right), k \geq 1$ by

$$
p_{k}\left(x_{1}, \ldots, x_{l} \mid \mathbf{u}\right)=\sum_{i=1}^{l}\left[x_{i}^{k}-u_{-i}^{k}\right] .
$$

Then the power sum function $p_{k}$ equals to the $k$-dimensional component of the equivariant Chern character of the difference bundle $\mathcal{E}_{l}-\underline{\mathcal{H}}_{-l,-1}$, i.e., $p_{k}\left(x_{1}, \ldots, x_{l} \mid \mathbf{u}\right)=$ $\operatorname{ch}_{k}^{T}\left(\mathcal{E}_{l}-\underline{\mathcal{H}}_{-l,-1}\right)$. Since $\operatorname{ch}^{T}\left(\mathcal{E}_{l}-\underline{\mathcal{H}}_{-l,-1}\right)=\operatorname{ch}^{T}\left(\mathcal{E}_{l, \text { even }}-\mathcal{F}_{l, \text { even }}\right)+\operatorname{ch}^{T}\left(\mathcal{E}_{l, \text { odd }}-\mathcal{F}_{l, \text { even }}\right)$, we find that

$$
\rho^{*} p_{k}\left(x_{1}, \ldots, x_{l} \mid \mathbf{u}\right)=p_{k}\left(x_{2}, x_{4} \cdots, x_{2[l / 2]} \mid \mathbf{u}_{\text {even }}\right)+p_{k}\left(x_{1}, x_{3}, \ldots, x_{2[(l-1) / 2]+1} \mid \mathbf{u}_{\text {odd }}\right) .
$$

Note that the function (5.1) can be extended to an element of the ring $\Lambda(x \| \mathbf{u})$ (to a symmetric function on $\tilde{R})$. More precisely, the formula

$$
p_{k}(x \mid \mathbf{u})=\sum_{i=1}^{\infty}\left[x_{i}^{k}-u_{-i}^{k}\right]
$$

is a finite sum on $\tilde{R}$ and therefore specifies an element of $\Lambda(x \| \mathbf{u})$. This formula is also equivalent to (1.10) under a change of variables. By (5.2) and (5.3), we obtain the following formula:

$$
\rho^{*} p_{k}(x \mid \mathbf{u})=p_{k}\left(x_{\text {even }} \mid \mathbf{u}_{\text {even }}\right)+p_{k}\left(x_{\text {odd }} \mid \mathbf{u}_{\text {odd }}\right),
$$

where $\left(x_{\text {even }}\right)_{k}=x_{2 k}$ and $\left(x_{\text {odd }}\right)_{k}=x_{2 k+1}$ for $k \in \mathbb{Z}$.

Let $\psi_{\text {even }}$ and $\psi_{\text {odd }}$ be the algebra isomorphisms $\Lambda\left(x_{\text {even }} \| \mathbf{u}_{\text {even }}\right) \rightarrow \Lambda(x \| \mathbf{u})$ and $\Lambda\left(x_{\text {odd }} \| \mathbf{u}_{\text {odd }}\right) \rightarrow \Lambda(x \| \mathbf{u})$ defined by $\psi_{\text {even }}\left(x_{2 k}\right)=x_{k}, \psi_{\text {even }}\left(u_{2 k}\right)=u_{k}$ and by $\psi_{\text {odd }}$ $\left(x_{2 k+1}\right)=x_{k}, \psi_{\text {even }}\left(u_{2 k+1}\right)=u_{k}$ for $k \in \mathbb{Z}$, respectively. Then $\psi_{\text {even }} \otimes \psi_{\text {odd }}$ determines an isomorphism from $H_{\mathbb{T}}^{*}\left(\operatorname{Gr}_{0}\left(\mathcal{H}_{\text {even }}\right)\right) \otimes H_{\mathbb{T}}^{*}\left(\operatorname{Gr}_{0}\left(\mathcal{H}_{\text {odd }}\right)\right)$ to $H_{\mathbb{T}}^{*}\left(\operatorname{Gr}_{0}(\mathcal{H})\right) \otimes H_{\mathbb{T}}^{*}\left(\operatorname{Gr}_{0}(\mathcal{H})\right)$. Combining the maps $\rho^{*}$ and $\psi_{\text {even }} \otimes \psi_{\text {odd }}$ we obtain a homomorphism

$$
\Delta: H_{\mathbb{T}}^{*}\left(\operatorname{Gr}_{0}(\mathcal{H})\right) \rightarrow H_{\mathbb{T}}^{*}\left(\operatorname{Gr}_{0}(\mathcal{H})\right) \otimes H_{\mathbb{T}}^{*}\left(\operatorname{Gr}_{0}(\mathcal{H})\right)
$$

so that (1.11) holds by (5.4). This completes the proof of the Theorem 1.5.

\section{Acknowledgments}

Both authors thank Max-Planck Institute für Mathematik in Bonn for generous support and wonderful environment. The second author was partially supported by the NSF grant no. DMS-0805989. 


\section{References}

[1] W. Fulton, Equivariant cohomology in algebraic geometry, Lecture Notes by D. Anderson (2007).

[2] M. Goresky, R. Kottwitz and R. MacPherson, Equivariant cohomology, Koszul duality, and the localization theorem, Invent. Math. 131(1) (1998), 25-83.

[3] I.G. Macdonald, Symmetric functions and Hall polynomials, Clarendon Press, Oxford, 2nd ed., 1995.

[4] G. Kempf and D. Laksov, The determinantal formula of schubert calculus, Acta Math. 132 (1974), 153-162.

[5] A. Knutson and T. Tao, Puzzles and (equivariant) cohomology of Grassmannians, Duke Math. J. 119(2) (2003), 221-260.

[6] J. Liou and A. Schwarz, Moduli spaces and Grassmannian, arXiv:1111.1649.

[7] J. Liou and A. Schwarz, Weierstrass cycles in moduli spaces and the Krichever map, arXiv: 1207.5030 .

[8] A.I. Molev and B. Sagan, A Littlewood-Richardson rule for factorial Schur functions, Trans. Amer. Math. Soc. 351(11) (1999), 4429-4443.

[9] A.I. Molev, Comultiplication rules for the double Schur functions and Cauchy identities, Electron. J. Comb. 16(1) Research Paper 13, 44pp (2009).

[10] A. Okounkov, G. Olshanski, Shifted Schur functions, St. Petersburg Math. J. 9 (1998), 239-300.

[11] A. Pressley and G. Segal, Loop groups, Oxford Mathematical Monographs. Oxford Science Publications. The Clarendon Press, Oxford University Press, New York (1986).

[12] A. Schwarz, Grassmannian and String theory, Comm. Math. Phys. 199(1), (1998), 1-24.

Max Planck Institute for Mathematics, Vivatsgasse 7, 53111 Bonn, Germany

E-mail address: fjmliou@mpim-bonn.mpg.de

Present Address: Einstein Institute of Mathematics, Edmond J. Safra Campus, Givat Ram, The Hebrew University of Jerusalem, 91904, Israel

Department of Mathematics, University of California, Davis, CA 95616, USA

E-mail address: schwarz@math.ucdavis.edu 